\title{
Partial nonlinear reciprocity breaking through ultrafast dynamics in a random photonic medium.
}

\author{
Otto L. Muskens, ${ }^{1}$ Paul Venn, ${ }^{1}$ Timmo van der Beek, ${ }^{2}$ and Thomas Wellens ${ }^{3}$ \\ ${ }^{1}$ School of Physics and Astronomy, University of Southampton, \\ Highfield, Southampton SO17, 1BJ, United Kingdom* \\ ${ }^{2}$ FOM Institute AMOLF, Science Park 104, 1098 XG Amsterdam, The Netherlands \\ ${ }^{3}$ Physikalisches Institut der Albert-Ludwigs Universitt Freiburg, \\ Hermann-Herder Str. 3, D-79104 Freiburg, Germany
}

\begin{abstract}
We demonstrate that ultrafast nonlinear dynamics gives rise to reciprocity breaking in a random photonic medium. Reciprocity breaking is observed via the suppression of coherent backscattering, a manifestation of weak localization of light. The effect is observed in a pump-probe configuration where the pump induces an ultrafast step-change of the refractive index during the dwell time of the probe light in the material. The dynamical suppression of coherent backscattering is reproduced well by a multiple scattering Monte Carlo simulation. Ultrafast reciprocity breaking provides a distinct mechanism in nonlinear optical media which opens up avenues for the active manipulation of mesoscopic transport, random lasers, and photon localization.

PACS numbers: 42.25.Bs, 78.47.J-, 78.67.Uh
\end{abstract}

There is a strong analogy between the transport of classical waves such as light and sound in scattering media, and the mesoscopic physics of quantum waves, such as electrons in a solid, or matter waves in an optical speckle potential [1]. Whereas in many quantum systems coherence is affected by inelastic processes, interactions are generally weak for light and coherent effects can be studied in large-scale systems. The role of interactions and decoherence in many-body quantum systems can be simulated by introducing a nonlinear optical response [2 7]. Recent pioneering experiments have explored nonlinear light scattering in Kerr-media and cold atomic clouds in the stationary regime [8 11].

In this Letter, we demonstrate partial breaking of the reciprocity symmetry in a random scattering medium on ultrafast time scales through the dynamics induced by a high-intensity, femtosecond pump pulse. As a probe for the breaking of reciprocity we use the coherent backscattering effect, a manifestation of weak localization for optical waves. Coherent backscattering (CBS) is the constructive interference of reciprocal light paths, resulting in a cone of enhanced intensity around the backscattering direction 12. The center of the cone results from the constructive interference of very long light paths, which makes CBS a sensitive probe for multiple scattering wave transport.

The reciprocity breaking effect is induced through ultrafast dephasing of light paths occurring in strongly scattering nanostructured semiconductors [13]. Reciprocity breaking requires the dynamics to be fast on the time scale of the photon dwell time in order to achieve an asymmetry between the direct and reciprocal paths. Such a regime of adiabatic control over light has been achieved only recently in high-quality photonic crystal nanocavities [14, 15], and is of interest for applications in controlled storage and release of optical information. Op- tical devices exploiting concepts from mesoscopic transport are currently receiving increasing interest [16 19]. A degree of active control over the flow of light in these devices would be of interest for applications.

The reciprocity breaking was measured in a slab of porous $\mathrm{GaP}$ of $81 \pm 2 \mu \mathrm{m}$ thickness, obtained by etching of a $\mathrm{GaP}$ wafer. Pump-probe nonlinear CBS cones were measured in a beamsplitter configuration as shown in Fig. 1(a). The frequency-doubled output from a regeneratively amplified Ti:Sapphire laser (Coherent RegA, $200 \pm 10 \mathrm{fs}, 250 \mathrm{kHz}$ ) at $400 \mathrm{~nm}$ wavelength was used as a pump for inducing an ultrafast nonlinear response. As a probe the signal output from a parametric amplifier at $630 \mathrm{~nm}$ wavelength was used. Both beams were focused to a $25-\mu \mathrm{m}$ diameter spot onto the sample using a lens with a focal length of $15 \mathrm{~cm}$ to achieve a sufficient pump fluence for nonlinear modulation, resulting in an angular resolution of $20 \mathrm{mrad}$. Backscattered light was collected using a Si-photodiode with a 0.5 -mm aperture, mounted on a translation stage positioned $6.5 \mathrm{~cm}$ away from the sample. Linear polarization filters were used to select either the polarization conserving $\left(R_{/ /}\right)$or the nonconserving $\left(R_{\perp}\right)$ scattering channels. The diffuse and CBS intensities $R_{\perp}$ and $R_{/ / /}$, as well as the differential pump-probe signals $\Delta R_{\perp}$ and $\Delta R_{/ /}$, i.e. the difference of the probe signal in presence of the pump with respect to $R_{\perp}$ or $R_{/ /}$, respectively, were measured using lock-in detection. All signals were normalized to the diffuse reflectance $R_{\perp}$ to divide out the angle-dependence of the setup and the (Lambertian) diffuse intensity distribution.

For the interpretation of our experimental results, Monte Carlo (MC) numerical simulations of the transient response were produced by spatiotemporal tracking of photon trajectories in a finite slab. Monte Carlo is a technique suitable for simulating diffuse transport and coherent backscattering of light [20]. In the current 
(a)

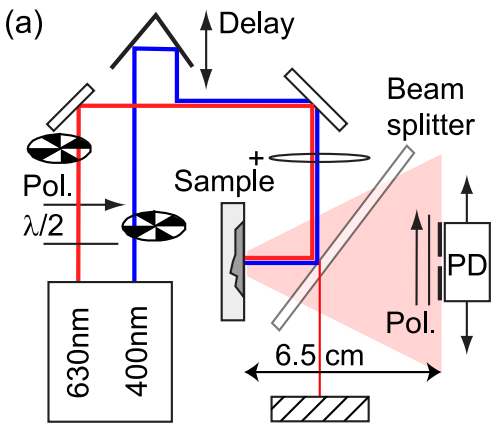

(b)

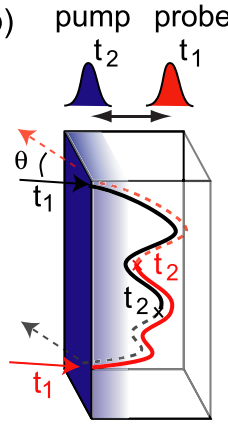

FIG. 1: (a) Experimental setup for time-resolved pump-probe coherent backscattering spectroscopy. (b) Principle of reciprocity breaking, where the symmetry of reciprocal multiple scattering path (arrows) is changed by a delayed pump pulse $\left(t_{2}>t_{1}\right)$.

model, illustrated in Fig. 1(b), the pump pulse induces a change in the complex refractive index at a time $t_{2}$. In all simulations in this Letter, we used one set of optimized parameters. These include the a-priory known parameters of sample thickness, laser pulse width and instrumental resolution. An internal reflection probability of 0.6 , corresponding to a linear refractive index $n_{0}=1.47$, was included, following measurements on similar porous GaP slabs 21]. Parameters extracted from comparison between numerical and experimental data (shown below) include the (linear) transport mean free path $\ell_{e}=0.45 \pm 0.1 \mu \mathrm{m}$ (corresponding to $k_{0} \ell_{e}=4.5$, with $k_{0}=2 \pi / 630 \mathrm{~nm}^{-1}$ the wavevector of the probe light in vacuum) and the (linear) transport mean free time $t_{e}=5 \pm 1 \mathrm{fs}$, the spatiotemporal refractive index $n(z, t)=n_{0}+\Delta n(z, t)$, where the nonlinear index change $\Delta n\left(z, t_{2}\right)=\left(1.4 \times 10^{-2}+1.4 \times 10^{-3} i\right) \exp \left(-z / L_{\text {exc }}\right)$ is contained within a photo-excited surface region of width $L_{\mathrm{exc}}=0.2 \mu \mathrm{m}$ just after absorption of the pump pulse at time $t_{2}$. The imaginary part of $\Delta n$ describes photoinduced absorption of the probe light, with absorption time $\tau_{\text {abs }}=n_{0} /\left(2 c k_{0} \operatorname{Im} \Delta n\right)=0.17$ ps at $z=0$ and time $t_{2}$. Subsequent spatiotemporal dynamics involve a rapid, picosecond expansion of the excited volume due to carrier migration, with a concomitant decrease in the nonlinear refractive index change $\Delta n$, such that the total amount of excitation in the volume remains unchanged by this expansion. In addition, the excitation is assumed to decay with a time constant of $30 \mathrm{ps}$, which, however, hardly affects the results presented in this Letter.

Figure 2(a) shows time-resolved reflectivity changes $\Delta R_{\perp} / R_{\perp}$ obtained in the cross-polarized, diffuse scattering channel. The signal at positive pump-probe time delays is characteristic for the excited semiconductor material and resembles our earlier measurements for bulk GaP (dash-dotted line) 13]. At negative delay times, an additional tail is present for the scattering layer. This tail corresponds to the contribution from photons enter-

(a)

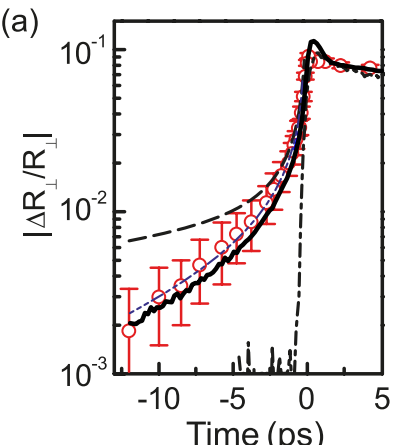

(c)
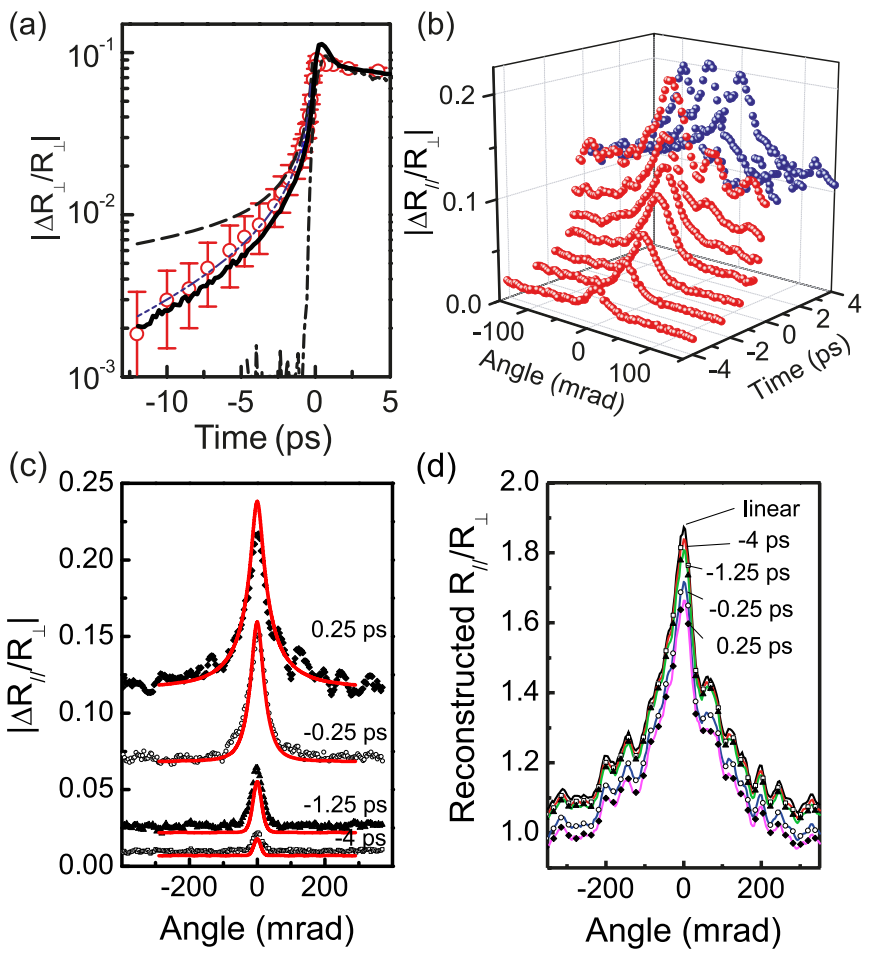

(d)

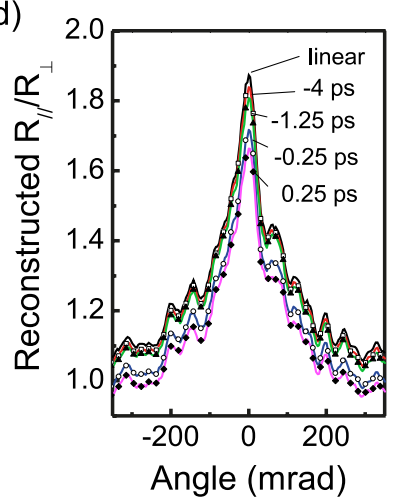

FIG. 2: (a) Differential diffuse reflectivity $\Delta R_{\perp} / R_{\perp}$ as a function of the time delay between pump and probe pulse. Lines indicate model calculations $\propto t^{-1 / 2}$ (dash), same but including an exponential cutoff with time-constant $10.2 \pm 0.7 \mathrm{ps}$ (dotted, blue), and Monte Carlo model (thick line). Dashdotted line: signal from a homogeneous (i.e. non-disordered) GaP substrate. (b) Angle- and time-resolved differential reflectivity $\Delta R_{/ /} / R_{\perp}$ in the polarization conserving channel, for negative (red) and positive (blue) time delays $t_{1}-t_{2}$. (c) Same as (b) for several delay times, including results from MC model (red lines). (d) CBS intensity traces without pump ('linear') and with pump reconstructed using the differential response curves from (c).

ing the sample before the pump and retained inside the sample for thousands of scattering events before undergoing photo-induced absorption initiated by the pump pulse.

The negative-time tail of Fig. 2(a) thus acts as a probe of the distribution of photon dwell times in the scattering medium, complementary to time-resolved interferometry experiments [22, 23]. Generally, the pump-probe signal can be expressed as a convolution of the diffuse pathlength distribution $\alpha_{d}(t)$ and a photo-induced absorption term $F(t)$, yielding

$$
\frac{\Delta R_{\perp}(t)}{R_{\perp}}=\frac{1}{\alpha_{d}^{\mathrm{tot}}} \int_{0}^{\infty} d t_{2} \alpha_{d}\left(t_{2}-t\right) F\left(t_{2}\right)
$$

where $t=t_{1}-t_{2}$ is the pump-probe delay time. The total albedo $\alpha_{d}^{\text {tot }}$ results from integrating $\alpha_{d}(t)$ over time. In the diffusive limit, the path length distribution is given by $\alpha_{d}(t) \propto t^{-3 / 2}$ for times larger than the extinction mean free time $t_{e}$ [24]. For the simple case of diffusion and a 


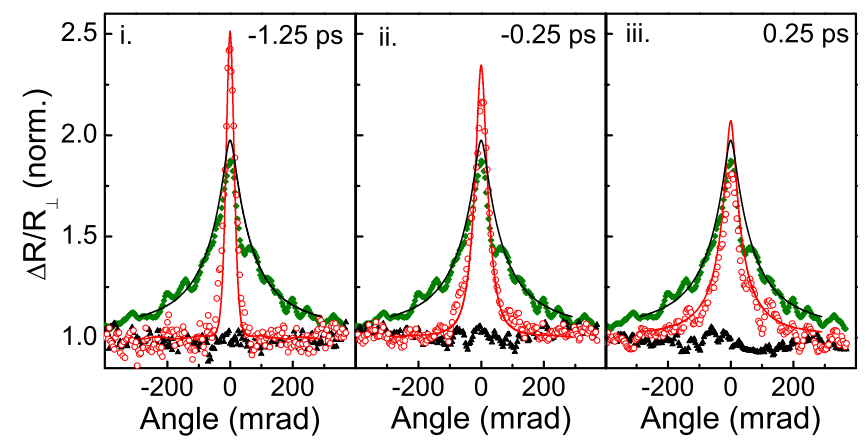

FIG. 3: Differential reflectivity for parallel (open circles, red) and cross-polarized (closed triangles, black) channels normalized to their values at $\theta=-270 \mathrm{mrad}$. Closed diamonds, green: Linear coherent backscattering cones. Lines: results from Monte Carlo model for linear and differential reflectivity cones. For negative delay times, the differential reflectivity in the parallel channel is larger than 2 , which indicates the occurence of partial reciprocity breaking.

step response $F(t)=\eta_{0} \Theta(t)$, Eq. (11) can be solved analytically, yielding $\Delta R_{\perp}(t) / R_{\perp}=\eta_{0}\left|t / t_{e}\right|^{-1 / 2}$ for $t<-t_{e}$. This relation is shown by the dashed line in Fig. 2 (a). Clearly, this curve overestimates the contribution of long paths in the tail for $t<-2$ ps. This discrepancy can be corrected by including an exponential cutoff in the distribution $\alpha_{d}(t)$ with time constant of $10.2 \pm 0.7 \mathrm{ps}$ corresponding to $\sim 2 \times 10^{3}$ scattering events [dotted line (blue) in Fig. 2(a)]. The loss of long light paths in the pump-probe signal is related to the finite system size as explained below. Our measurements show that pumpprobe transient absorption forms a sensitive probe of very long light paths in the photonic medium.

The above form of $F(t)$ is valid under the assumption that only a thin surface layer is affected by the pump pulse, which is a good approximation given the bulk absorption length of $\mathrm{GaP}$ of around $0.2 \mu \mathrm{m}$ at $400 \mathrm{~nm}$ wavelength 25]. Therefore, the relative amount of absorption is approximately constant $\left(\eta_{0} \simeq 10^{-1}\right)$ for all probe photons which undergo a scattering sequence whose length exceeds the pump-probe delay time $t$. This picture is confirmed by the MC model (thick solid line). It was found from the MC model that the slope of the negative-time tail is determined entirely by the $25-\mu \mathrm{m}$ transverse spot size of the pump beam. Long light paths diffuse out of the excited region, resulting in the observed reduction of the long-time tail. The spatiotemporal expansion mainly affects the decay at positive times in Fig. 2(a). The real part of $\Delta n$ does not affect the diffuse tail; however it is mainly responsible for the reciprocity breaking effect as shown below.

We proceed to investigate the effect of ultrafast excitation on the CBS intensity in the polarization conserving channel. Figure 2(b) shows the experimental differential reflectivity $\Delta R_{/ /} / R_{\perp}$. We emphasize that these nonlinear CBS traces are obtained as the difference between

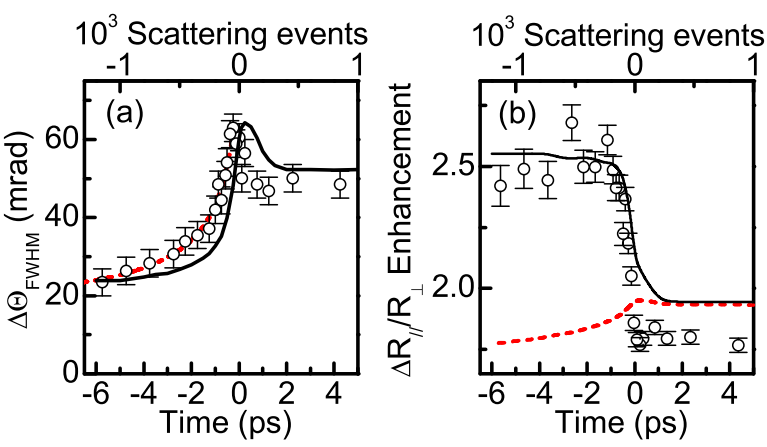

FIG. 4: (a) Full width at half maximum values of the CBS cone width, with calculation using the CBS albedo Eq. (2) (dashed line, red) and Monte Carlo model (thick line). (b) Experimental pump-probe enhancement factors (circles) in comparison with the Monte Carlo model for the full, complex nonlinear refractive index as described in text (line) and using only the imaginary part of $\Delta n$ (dashed line, red).

two CBS intensity cones respectively in presence and absence of the pump pulse, as is typical for lock-in detection. Therefore, the differential reflectivity corresponds to the intensity which is removed from the linear CBS cone as a consequence of photo-induced effects. This can be observed in Fig. 2(c,d) where we have used the differential signals to reconstruct the modified intensity CBS cones at various delay times. The cone rounds off and effectively broadens as long light paths are removed. Both the coherent backscattering and the diffuse background show a maximum decrease of around $12 \%$ around the pump-probe delay of $0 \mathrm{ps}$.

The contribution of reciprocity breaking to the overall pump-probe signal can be analyzed by comparing the ratio of of the intensities removed from the CBS with those removed from the diffuse albedo. In particular, the more the total CBS cone decreases due to reciprocity breaking, the more the differential CBS cone increases, which may thus assume a relative height of larger than 2. Figure 3 shows the three differential CBS cones i, ii, iii of Fig.2(c) (dots, red), where the change in the diffuse background at $-270 \mathrm{mrad}$ is normalized to 1 . For comparison, also the diffuse pump-probe signals are shown (triangles, black). In comparison to the linear CBS intensity cone (diamonds, green), the differential pump-probe cones are narrower and have a higher amplitude. The values for the angular width and differential cone height are shown in Fig. 4(a,b) against pump-probe delay time.

To understand the trends in Fig. 3]and 4 , we start from the characteristic time- and angle-dependent albedo of the CBS cone given by 24]

$$
\alpha_{c}(\theta, t) \simeq \alpha_{d}(t) e^{-\frac{1}{3}\left(k_{0} \ell_{e} \theta\right)^{2} t / t_{e}},
$$

with $\theta$ the backscattering angle. At exact backscattering, Eq. (2) reduces to the diffuse albedo, $\alpha_{c}(0, t)=\alpha_{d}(t)$. The angular range $\theta$ over which paths of a characteristic 
time duration $\tau_{C}$ contribute to the CBS-cone is given by $\theta\left(\tau_{C}\right)=\left(3 t_{e} / k_{0}^{2} \ell_{e}^{2} \tau_{C}\right)^{1 / 2}$. This relation shows that the angular width is reduced proportional to the square root of the path time duration. At long negative delay times $t=t_{1}-t_{2}$, only long light paths with dwell times exceeding $|t|$ contribute to the nonlinear signal, and hence $\tau_{C}=|t|$. This relationship - which clearly explains the fact that the nonlinear CBS traces are narrower than the linear ones, as already observed in Fig. 3 - is indicated by the dashed line (red) in Fig. 4(a). The calculated curve includes the instrumental resolution of $20 \mathrm{mrad}$, and was fitted to the experimental data at negative delay times in order to determine $t_{e}$. Around $t=0$, the cone width exhibits a maximum, which is reproduced by the MC simulation (thick solid line) and can be attributed to the spatio-temporal dynamics of the excited surface region after excitation. In the $\mathrm{MC}$ simulation, the latter is modelled as an expansion of the excited surface from $L_{\text {exc }}=0.2 \mu \mathrm{m}$ to $2.25 \mu \mathrm{m}$ within a time of $2 \mathrm{ps}$. Due to this expansion, the size of the excited region encountered by the probe photons increases for positive delay times, leading to stronger absorption of long scattering paths, and consequently, to a narrower differential cone.

The maximum height of the $\Delta R_{/ /} / \Delta R_{\perp}$ enhancement factor is shown against delay time in Fig. 4(b). If this factor is larger than 2, this indicates that relatively more intensity is removed from the CBS- than from the diffuse scattering-albedo. Figure 4(b) shows that this additional contribution is only present at negative delay times, i.e. for probe photons arriving before the pump pulse. This behavior is reproduced by our MC simulations, and can be attributed to the asymmetry between direct and reciprocal light paths induced by the nonlinear refractive index change [26]. Only changes fast with respect to the photon dwell time are able to break reciprocity. Such fast changes are mainly obtained at the leading edge of the ultrafast nonlinear response of GaP. As long as the probe photons arrive before this nonlinear edge, a sufficient reciprocity breaking is obtained to produce an effect in the nonlinear CBS-enhancement. Given the maximum value of 2.5 for the $\Delta R_{/ /} / \Delta R_{\perp}$ ratio and our observation that the diffuse scattering albedo of long light paths is suppressed by $\eta_{0} \simeq 10 \%$, we conclude that the CBS albedo of these light paths is reduced, on average, by approximately $15 \%$, corresponding to a partial reciprocity breaking of about $5 \%$.

Dephasing and absorption both result in an asymmetric reduction of the amplitudes, and thus a reciprocity breaking effect. In the MC simulation (line) in Fig. 4(b), good agreement with experiments is found when assuming for the real part to be ten times larger than the imaginary part of $\Delta n$. This implies that the effect of dephasing is much stronger, consistent with our earlier results [13]. A purely imaginary $\Delta n$ (dashed line) provides only a small reciprocity breaking effect which cannot explain the experimental enhancement factors.
In conclusion, we have demonstrated partial suppression of reciprocity in a random medium through the coherent backscattering effect. Reciprocity is a fundamental property of photonic media, and its ultrafast control opens up a new approach to nonlinear manipulation of photonic eigenstates. Our work is the first step toward the control of mesoscopic interference phenomena in random media. We achieve $5 \%$ reciprocity breaking using only a $200-n m$ thin excitation region. Typically, localization lengths in random media and gain lengths in random lasers amount to at least several micrometers [1, 18]. Ultrafast reciprocity breaking of closed loops and random laser modes is therefore feasible but requires further optimization of the pumping conditions to achieve dephasing in a large excitation volume. This may be achieved using a longer pump absorption length, two-photon absorption, or use of two-dimensional waveguides where pump light can be coupled in from the top of the structure.

We thank the FASTlab and D. Kundys for support, and A. Lagendijk for stimulating discussions. This work was supported by EPSRC through grant EP/J016918/1 and by DFG through grant BU 1337/8-1.

* Electronic address: O.Muskens@soton.ac.uk

[1] A. Lagendijk, B. van Tiggelen, D. S. Wiersma, Physics Today 62, 24 (2009).

[2] I. V. Shadrivov, K. Y. Bliokh, Yu. P. Bliokh, V. Freilikher, and Yu. S. Kivshar, Phys. Rev. Lett. 104, 123902 (2010).

[3] T. Wellens and B. Gremaud, Phys. Rev. Lett. 100, 033902 (2008).

[4] B. Spivak, A. Zyuzin, Phys. Rev. Lett. 84, 1970 (2000).

[5] S. E. Skipetrov and R. Maynard, Phys. Rev. Lett. 85, 736 (2000).

[6] C. Conti, L. Angelani, and G. Ruocco, Phys. Rev. A 75, 033812 (2007).

[7] C. Conti and L. Leuzzi, Phys. Rev. B 83, 134204 (2011).

[8] T. Schwartz, G. Bartal, S. Fishman, and M. Segev, Nature 446, 52 (2007).

[9] U. Bortolozzo, S. Residori, and P. Sebbah, Phys. Rev. Lett. 106, 103903 (2011).

[10] G. Labeyrie, Mod. Phys. Lett. B 22, 73 (2008).

[11] D. Wilkowski, Yannick Bidel, T. Chaneliere, R. Kaiser, B. Klappauf, C. Miniatura, SPIE Proc. Opt. Eng. 5866, 298 (2005).

[12] M. P. Van Albada and A. Lagendijk, Phys. Rev. Lett. 55, 2692; P.-E. Wolf and G. Maret, Phys. Rev. Lett. 55, 2696 (1985).

[13] M. Abb, E.P.A.M. Bakkers, O. L. Muskens, Phys. Rev. Lett. 106, 143902 (2011).

[14] M. Notomi, Rep. Prog. Phys. 73, 096501 (2010).

[15] G. Ctistis, E. Yuce, A. Hartsuiker, J. Claudon, M. Bazin, J.-M. Grard, and W. L. Vos, Appl. Phys. Lett. 98, 161114 (2011).

[16] E.G. van Putten, D. Akbulut, J. Bertolotti, W.L. Vos, A. Lagendijk, and A.P. Mosk, Phys. Rev. Lett. 106, 193905 (2011). 
[17] G. Lerosey, J. de Rosny, A. Tourin, and M. Fink, Science 315, 1120 (2007).

[18] D. S. Wiersma, Nat. Phys. 4, 359 (2008).

[19] L. Sapienza, H. Thyrrestrup, S. Stobbe, P. David Garcia, S. Smolka, and P. Lodahl, Science 327, 1352 (2010).

[20] J. Sawicki, N. Kastor, M. Xu, Opt. Expr. 16, 5728, (2008).

[21] J. G. Rivas, D. H. Dau, A. Imhof, R. Sprik, B. P. J. Bret, P. M. Johnson, T. W. Hijmans, and A. Lagendijk Optics Commun. 220, 17-21 (2003).

[22] P. M. Johnson, A. Imhof, B. P. J. Bret, J. G. Rivas, and A. Lagendijk, Phys. Rev. E 68, 016604 (2003).

[23] C. Toninelli, E. Vekris, G. A. Ozin, S. John, and D.
S. Wiersma, Phys. Rev. Lett. 101, 123901 (2008); R. Sapienza, P. Costantino, D. Wiersma, M. Ghulinyan, C. J. Oton, and L. Pavesi, Phys. Rev. Lett. 91, 263902 (2003).

[24] E. Akkermans and G. Montambeaux, Mesoscopic physics of electrons and photons (1st ed. Cambridge, 2007).

[25] E.D. Palik. Handbook of Optical Constants, Academic Press, New York (1985). (1998). (2010).

[26] The difference in linear and nonlinear CBS enhancement factors between MC simulations and experiments results from a reduced experimental CBS enhancement factor for the linear cone which was not included in the model. 